\title{
A low caffeine dose improves maximal strength, but not relative muscular endurance in either heavier- or lighter- loads, or perceptions of effort or discomfort at task failure in females
}

\author{
Georgina Waller ${ }^{1}$, Melissa Dolby ${ }^{1}$, James Steele ${ }^{1}$, James P Fisher ${ }^{\text {Corresp. } 1}$ \\ ${ }^{1}$ Sport, Health and Social Sciences, Southampton Solent University, Southampton, United Kingdom \\ Corresponding Author: James P Fisher \\ Email address: james.fisher@solent.ac.uk
}

Background. The body of literature considering caffeine as an ergogenic aid has primarily considered typically aerobic based exercise, male participants and moderate- to largecaffeine doses. With this in mind the aim of this project was to explore the effects of a lowcaffeine dose upon maximal voluntary contraction (MVC), and muscular endurance (time to task failure; TTF) at heavier- and lighter-loads. Methods. Nineteen physically active, habitual caffeine consuming females randomly performed 4 testing conditions; 2 with a low-dose of caffeine $\left(100 \mathrm{mg}\right.$ equating to mean $\left.=1.5 \pm 0.18 \mathrm{mg} \cdot \mathrm{kg}^{-1}\right)$ and 2 placebo conditions, where they performed a maximal strength test (MVC) knee extension at $45^{\circ}$ followed by a task of relative muscular endurance (sustained isometric contraction for time to task failure; TTF) using either heavier- (70\% MVC) and lighter- (30\% MVC) loads. Each participant performed each load condition following both caffeine and placebo consumption. Immediately following cessation of the muscular endurance test participants were asked to report their rating of perceived effort (RPE) and rating of perceived discomfort (RPD). Results. Analyses revealed a significant effect for caffeine upon MVC compared to placebo $(p=0.007)$. We also found a significantly greater TTF for the lightercompared to the heavier-load condition $(p<0.0001)$; however, there was no significant effect comparing caffeine to placebo $(p=0.2368)$, but insufficient precision of estimates to infer equivalence in either lighter- $(p=0.750)$ or heavier-load $(p=0.262)$ conditions. There were no statistically significant effects for caffeine compared with placebo, or lightercompared with heavier-loads, for RPE and RPD (all $p>0.05$ ). RPE was statistically equivalent between caffeine and placebo for both lighter- $(p=0.007)$ and heavier-load $(p=0.002)$ conditions and RPD for heavier- $(p=0.006)$ but not lighter-load ( $p=0.136)$.

Discussion. This is the first study to demonstrate a positive effect on strength from a low caffeine dose in female participants. However, it is unclear whether caffeine positively 
impacts upon relative muscular endurance in either heavier- or lighter-loads. Further, both RPE and RPD appear to be relatively similar during isometric tasks performed to task failure independently of caffeine supplementation or load. These findings may have implications for persons wishing to avoid side-effects or withdrawal symptoms associated with larger caffeine doses whilst still attaining the positive strength responses. 
1 A low caffeine dose improves maximal strength, but not relative muscular endurance in either heavier- or lighter-

2 loads, or perceptions of effort or discomfort at task failure in females

3

$4 \quad$ Running Title: Low caffeine dose improves strength in females

5

6 Original Research Article

7

8 Georgina Waller ${ }^{1}$, Melissa Dolby ${ }^{1}$, James Steele ${ }^{1}$, James Peter Fisher ${ }^{1}$

9

10 'Solent University, East Park Terrace, Southampton, UK

11

12 Corresponding Author:

13 James Fisher

14 Solent University, East Park Terrace, Southampton, UK

15 Tel: +442382013163

16 Email: James.Fisher@solent.ac.uk

17

18 Co-Author contact details:

19 Georgina Waller (georgie9809@aol.com)

20 Melissa Dolby (melissadolby95@gmail.com)

21 James Steele (James.steele@solent.ac.uk)

22

23 Word Count: 5675

24 Abstract: 357 
25

26

27

28

29

30

31

32

33

34

35

36

37

38

39

40

41

42

43

44

45

46

47

48

49

\section{Abstract}

Background. The body of literature considering caffeine as an ergogenic aid has primarily considered typically aerobic based exercise, male participants and moderate- to large-caffeine doses. With this in mind the aim of this project was to explore the effects of a low-caffeine dose upon maximal voluntary contraction (MVC), and muscular endurance (time to task failure; TTF) at heavier- and lighter-loads.

Methods. Nineteen physically active, habitual caffeine consuming females randomly performed 4 testing conditions; 2 with a low-dose of caffeine (100mg equating to mean $=1.5 \pm 0.18 \mathrm{mg} \cdot \mathrm{kg}^{-1}$ ) and 2 placebo conditions, where they performed a maximal strength test (MVC) knee extension at $45^{\circ}$ followed by a task of relative muscular endurance (sustained isometric contraction for time to task failure; TTF) using either heavier- (70\% MVC) and lighter- (30\% MVC) loads. Each participant performed each load condition following both caffeine and placebo consumption. Immediately following cessation of the muscular endurance test participants were asked to report their rating of perceived effort (RPE) and rating of perceived discomfort (RPD).

Results. Analyses revealed a significant effect for caffeine upon MVC compared to placebo ( $p=0.007)$. We also found a significantly greater TTF for the lighter-compared to the heavier-load condition $(p<0.0001)$; however, there was no significant effect comparing caffeine to placebo $(p=0.2368)$, but insufficient precision of estimates to infer equivalence in either lighter- $(p=0.750)$ or heavier-load $(p=0.262)$ conditions. There were no statistically significant effects for caffeine compared with placebo, or lighter-compared with heavier-loads, for RPE and RPD (all $p>0.05)$. RPE was statistically equivalent between caffeine and placebo for both lighter- $(p=0.007)$ and heavierload $(p=0.002)$ conditions and RPD for heavier- $(p=0.006)$ but not lighter-load $(p=0.136)$.

Discussion. This is the first study to demonstrate a positive effect on strength from a low caffeine dose in female participants. However, it is unclear whether caffeine positively impacts upon relative muscular endurance in either heavier- or lighter-loads. Further, both RPE and RPD appear to be relatively similar during isometric tasks performed to task failure independently of caffeine supplementation or load. These findings may have implications for persons wishing to avoid side-effects or withdrawal symptoms associated with larger caffeine doses whilst still attaining the positive strength responses. 
50 Key words: strength, maximal voluntary contraction, isometric, discomfort, effort, ergogenic

51

52

53

54

\section{Introduction}

Caffeine is evidenced to increase productivity, improve concentration, and reduce fatigue (Bazzucchi et al. 2011; Pickering and Kiely, 2018) and as such, in an effort to enhance performance, it is reported that $74 \%$ of athletes consume caffeine as an ergogenic aid prior to competition (Del Coso et al. 2011). Historically the body of research has considered the effects of caffeine upon predominantly aerobic endurance type exercise, for example; time to exhaustion in well-trained and elite cyclists (Costill et al. 1978, Pasman et al. 1995), 2000m rowing performance (Bruce et al. 2000), and 1500m treadmill running (Wiles et al. 1992). Indeed, a recent umbrella review of published meta-analyses considering the effects of caffeine supplementation on a range of performance outcomes found that evidence was stronger for predominantly aerobic vs. anaerobic tasks (Grgic et al. 2019a). Yet, perhaps in part due to the popularity of caffeinated supplements aimed to harness its aforementioned benefits, caffeine is commonly consumed as part of a 'pre-workout' with the desire to enhance strength, power and muscular endurance before partaking in resistance exercise or strength/power-based tasks (Cesareo et al. 2019). In comparison with aerobic endurance tasks, there is still a gap within the present body of literature considering caffeine consumption and muscular strength/endurance tasks. Further, as with most resistance training studies, the body of research considering the maximal strength and muscular endurance effects of caffeine have dominantly considered trained or untrained male participants.

Indeed, a recommendation from recent reviews by Grgic et al. (2019a;2019b) was for more research to be conducted in women. This is supported by evidence suggesting sex-differences in subjective and physiological responses to caffeine consumption (Temple and Ziegler 2011). To date research considering female participants has used large caffeine doses. For example, Goldstein et al. (2010) considered 15 females, reporting a small yet significantly $(p<0.05)$ greater barbell bench press $1 \mathrm{RM}$ for caffeine $\left(6 \mathrm{mg} \cdot \mathrm{kg}^{-1}\right)$ compared to placebo conditions $(52.9$

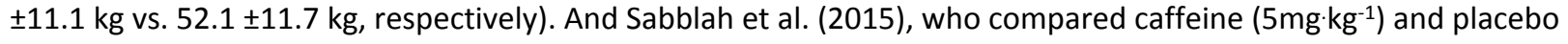
for strength differences between males $(n=10)$ and females $(n=8)$. The authors reported significantly greater 1RM bench press following caffeine compared to placebo, $(p=0.016$; males $=101.5 \pm 28.9$ to $107.5 \pm 30.5 \mathrm{~kg}$, females $=$ $32.2 \pm 9.0$ to $35.3 \pm 7.3 \mathrm{~kg}$ ) with no between sex differences. In addition, they reported a tendency for an increase in 
76

77

78

79

80

81

82

83

84

85

86

87

88

89

90

91

92

93

94

95

96

97

98

99

100

relative muscular endurance (repetitions at 40\% 1RM; $p=0.059$ ) with a between sex difference of $p=0.061$ in favour of males compared to females as a result of caffeine ingestion. Finally, Sabblah et al. (2015) found no improvement in squat $1 \mathrm{RM}$ for either males or females for the caffeine condition. The lack of research considering female participants might simply be a reflection that the bulk of strength training research considers male participants. However, it might also be a product of the sensitivity around female menstrual cycles and oral contraceptive use which might impact the effects of caffeine upon the body (Abernethy and Todd 1985).

A further limitation of present literature is a lack of investigation into low caffeine dosage $\left(<3 \mathrm{mg} \cdot \mathrm{kg}^{-1}\right)$ for strength measures (Spriet 2014). Indeed, Grgic et al. (2019a) note that the doses of caffeine across the body of literature have generally been around $6 \mathrm{mg}$ of caffeine per kilogram of body mass (mg. $\mathrm{kg}^{-1}$ ) or greater. Some studies suggest that lower doses of caffeine could be impactful upon strength performance. For example, Arazi et al. (2016) reported maximal strength (leg press $1 \mathrm{RM})$ across 2 caffeine conditions $\left(2 \mathrm{mg} \cdot \mathrm{kg}^{-1}\right.$ and $\left.5 \mathrm{mg} . \mathrm{kg}^{-1}\right)$ as well as a placebo condition. Whilst the analyses didn't reveal a statistically significant difference, their descriptive data (leg press $1 \mathrm{RM}$; placebo $=154 \pm 27.3 \mathrm{~kg}$ vs. $2 \mathrm{mg} . \mathrm{kg}^{-1}$ of caffeine $=171 \pm 15.9 \mathrm{~kg}$ ) seems noteworthy. A more recent study of the dose-response effects of $2 \mathrm{mg} \cdot \mathrm{kg}^{-1}, 4 \mathrm{mg} \cdot \mathrm{kg}^{-1-}$, and $6 \mathrm{mg} \cdot \mathrm{kg}^{-1}$ of caffeine upon both upper and lower body strength and relative muscular endurance in males reported a linear trend for upper body strength, but no clear dose-response relationship for lower body strength or relative muscular endurance (Grgic et al. 2019c). Thus, there is the possibility that low doses of caffeine may still produce performance enhancing effects. This might be particularly important considering studies reporting negative side effects to caffeine supplementation. For instance, Goldstein et al. (2010) reported that $20 \%$ of participants "exhibited intense emotional side effects, including an expressed inability to verbally communicate, focus, and/or remain still as well as the feeling of wanting to $c r y$ " (page 4). Other studies have negative side-effects following caffeine withdrawal, including severe fatigue, increased muscle pain and cramps, sleep disturbance, irritability, headaches, and occasional nausea (Nehlig 1999; Juliano and Griffiths 2004). In review, withdrawal symptoms were evident from a median dose of $357 \mathrm{mg}$ (in a $60 \mathrm{~kg}$ female and $80 \mathrm{~kg}$ male this equates to $5.95 \mathrm{mg} \cdot \mathrm{kg}^{-1}$ and $4.46 \mathrm{mg} \cdot \mathrm{kg}^{-1}$, respectively) but also in doses as low as $129 \mathrm{mg}$ (in the same example: $2.15 \mathrm{mg} \cdot \mathrm{kg}^{-1}$ and $1.61 \mathrm{mg} \cdot \mathrm{kg}^{-1}$, respectively; Strain et al. 1994). 

considered the impacts of caffeine supplementation upon the effects of heavier-compared with lighter-load muscular endurance tasks. These might differ following caffeine consumption as a result of the effects of caffeine on the neurological system and the potentiation of force production during submaximal intensities (Tarnopolsky 2008), as well as the potentially differing effects of caffeine on slow twitch muscle fibres (Davis and Green 2009). Current research suggests that lighter-load resistance exercise tasks, whilst producing similar perceptions of effort at momentary task failure, produce greater discomfort compared to heavier loads (e.g. Fisher and Steele 2017; Stuart et al. 2018). However, these studies did not consider the use of caffeine ingestion which might improve endurance performance through a reduction in perceived effort at submaximal exercise intensities thus deterring task disengagement due to maximal perceived effort for longer (Duncan et al. 2013; de Morree et al. 2014; level of pain perception, or again deterring task disengagement due to reaching maximum tolerable pain levels (Motl et al. 2003; Motl et al. 2006; Astorino et al. 2011; Duncan et al. 2013). Indeed, relative muscular endurance typically does not change with interventions including resistance training, but where it does it has been speculated this may be due to reductions in perceived discomfort (Fisher et al., 2020). However, both the perceived effort and perceived pain reducing effects of caffeine have only been shown with higher doses $\left(4-10 \mathrm{mg}^{\mathrm{kg}} \mathrm{kg}^{-1}\right)$. related to the perceptual effects of caffeine. 
126

128

130

131

134

135

136

137

138

140

141

142

\section{Experimental Approach to the Problem}

The present study used a double-blind, randomised control and crossover counterbalanced research design whereby all participants completed caffeine and placebo conditions for maximal strength testing (MVC) of the knee extensors and both heavier- (70\% MVC) and lighter- (30\% MVC) load isometric knee extension time to task failure (e.g. all participants performed 4 conditions).

\section{Participants}

Following approval from Solent University Health, Exercise and Sport Science ethics committee (ethical application reference number: wallg2018), 19 physically active female participants (age range $18-35$ years, mean ( \pm SD) age $=21.6 \pm 3.8$ years, height $=1.68 \pm 0.07 \mathrm{~cm}$, mass $=67.9 \pm 8.4 \mathrm{~kg}$, body mass index $=24.2 \pm 2.8)$ were recruited from a University campus. All participants were considered healthy based upon responses to a modified physical activity readiness questionnaire (PARQ). Inclusion criteria required that all participants be habitual caffeine drinkers (>100mg/day and recorded via a 4-day food diary based on Motl et al. 2006) and be free from musculoskeletal injuries for which a knee extension task might be contraindicated, as well as not currently undertaking a structured resistance training programme. All participants were provided a verbal briefing about the study, provided a participant information sheet for their records, and completed an informed consent form.

Exclusion criteria included being pregnant, breast feeding ( $\leq 6$ months), consuming androgenic anabolic steroids, diabetic, hypertensive, consuming $>300 \mathrm{mg} /$ day of caffeine.

Familiarisation

Prior to testing, each participant attended the laboratory where height and mass were recorded, and paperwork were completed. The isokinetic dynamometer (Humac Norm, CSMi, Stoughton, MA) was set up to ensure participant comfort, whilst the lateral epicondyle of the right knee was aligned with the axis of rotation on the device. The cushioning pad of the leg extension was positioned central to the shin and seat and position measurements were recorded for replication for each condition for each participant. All participants confirmed right leg dominance when asked with which leg they would kick a football with and thus, the right leg was used for testing for all conditions. Following set-up each participant completed MVC testing at $45^{\circ}$ of knee extension and 
151 the lighter load ( $30 \%$ MVC) condition as outlined below (data was not recorded) to provide familiarisation to

152 isokinetic testing and isometric time to task failure. During the familiarisation session participants were also

153 informed of the use of rating of perceived exertion-effort and rating of perceived exertion and perceived

154 discomfort scales (Steele et al. 2017). The scales and instructions are available on the Open Science Framework

155 (https://osf.io/ufvy8/).

156

157

158

159

160

161

162

163

164

165

166

167

168

169

170

171

172

173

\section{Blinding, Randomisation and Dosage}

A person external to the project was asked to label both the caffeine and placebo tablet as supplement ' $A$ ' or ' $\mathrm{B}$ ' and deposit each into an opaque container to ensure double blinding from both the researchers providing the supplement and the participants. The caffeine consisted of $2 \times$ Pro-Plus Tablets (a total of $100 \mathrm{mg}$ caffeine), and the placebo was $2 \times$ Deva Vegan D2 Vitamin D 1200 IU tablet (0.06mg D2). Vitamin D2 was selected based on similarity and size to the caffeine tablet and since studies have shown this to be ineffective upon muscular strength (Chiang et al. 2017). For the caffeine conditions the dosage of caffeine was maintained at 100mg per person since this represents ecological validity for consumption (e.g. we believe a person is likely to consume the dosage suggested on the packet not calculate a specific dosage based on their body mass). The dosage provided resulted in a body mass relative dose at $1.50 \pm 0.18 \mathrm{mg}^{\cdot \mathrm{kg}^{-1}}$ (range $=1.1$ to $\left.1.85 \mathrm{mg} \cdot \mathrm{kg}^{-1}\right){ }^{1}$

\section{Testing}

On the morning of testing, participants had been instructed not to consume caffeine-containing food or beverages. A sheet including drinks, food and medication to avoid was provided in advance to participants (in brief this included; coffee, tea, chocolate, carbonated beverages, energy drinks of any kind, caffeine/energy gels, chewing gum, supplement bars including protein and energy bars, weight-loss tablets, pain-relieving tablets including codeine, paracetamol, ibuprofen, etc., and alcohol). Participants confirmed they had not participated in strenuous exercise for 48 -hours prior to testing for each condition. Participants were not requested to attend the testing sessions in a fasted state but were asked to replicate any foods consumed prior to the first testing

\footnotetext{
${ }^{1}$ For context and comparison, an $8.4 \mathrm{fl} \cdot \mathrm{oz} / 250 \mathrm{ml}$ can of Red Bull contains $80 \mathrm{mg}$ of caffeine, equal to $1.17 \mathrm{mg} \cdot \mathrm{kg}^{-1} \mathrm{for}$ the present participants.
}

Peer) reviewing PDF | (2020:02:45680:1:0:NEW 6 Apr 2020) 
174

175

condition for the latter conditions. Participants attended the laboratory for testing between 8am and 11 am, and testing time was standardized for each participant across all conditions.

Participants returned no less than 6 days following the familiarisation for the first testing session to eliminate the effects of any delayed onset muscle soreness. Each participant received either supplement ' $A$ ' or ' $B$ ' (selected at random via a random letter generator) and was asked to consume it together with 150ml of tap water (Smit and Rogers 2000). After 60 minutes, during which no food consumption or physical activity was permitted, and toilet breaks and water consumption were logged, the participant was asked to complete a brief 5-minute warm-up on a cycle ergometer (Monark, Ergomedic 874e) up to $60 \%$ age-predicted maximum heart rate to warm key muscles (Fisher and Steele 2017). Following this, the participant completed a maximal voluntary contraction (MVC) at $45^{\circ}$ knee extension. Participants were instructed to gradually build up to a maximal effort over 3 seconds and were instructed to gradually reduce their effort once it was clear that a max torque had been achieved (i.e. when the torque reading was no longer increasing). After 30 seconds rest, the participant completed the isometric leg extension task to failure. The lever arm was maintained at $45^{\circ}$ knee extension and the participant was provided visual feedback on a display screen of what force to maintain (70\% or 30\% for heavier- and lighter-load conditions, respectively) with an upper and lower bar of $\pm 5 \%$ (e.g. if MVC was $100 \mathrm{Nm}$ then for the $70 \%$ condition a bar was shown at $70 \mathrm{Nm}$ with an upper and lower bar $3.5 \mathrm{Nm}$ above and below, respectively). Verbal encouragement was provided throughout to ensure maximal exerted effort (Timmins and Saunders 2014), and if they fell below the bottom torque limit then they were encouraged to attempt to regain their set torque output. The task was ceased when the participant could not maintain the required force despite attempting to with maximal effort. Rating of perceived effort (RPE) and rating of perceived discomfort (RPD) was measured and recorded immediately following the isometric knee extension task (Steele et al. 2017) along with time to task failure (TTF; seconds).

For the successive 3 testing sessions with no less than 6 days recovery between, participants repeated the testing process as described above. Each participant was randomly assigned a supplement and condition from their remaining allocation, e.g. each participant had $2 \times{ }^{\prime} A$ ' and $2 \times$ ' $B$ ' supplements, for both heavier- and lighterload conditions (70\% and 30\% MVC, respectively). 
200

201

202

203

204

205

206

207

208

209

210

211

212

213

214

215

216

217

218

219

220

221

222

223

224

225

\section{Statistical analysis}

The independent variables were the training load (70\% MVC and 30\% MVC) and the supplement condition (placebo and caffeine). The dependent variables included MVC, TTF, RPE, and RPD. All analysis was done using the "Ime4" package (Bates et al. 2015) and "ImerTest" in R (v3.6.1; R Foundation for Statistical Computing, Vienna, Austria. URL http://www.R-project.org/). Linear mixed modelling was used to analyse all outcomes using Restricted Maximum Likelihood estimation with participants as a level 3 variable, 'Load' (70\% MVC and 30\% MVC) as a level 2 variable, and 'Condition' (placebo and caffeine) as a level 1 variable. 'Load' and 'Condition' were modelled as fixed factors with random intercepts by participants included. For MVC only Condition as examined as a factor with 4 separate observations per participant and 2 per condition due to this outcome being measured at the beginning of every trial. Estimated marginal means with $95 \%$ confidence intervals were produced using the "emmeans" package from each model (for each Condition for MVC, and for each Condition by Load for all other dependent variables) in addition to pairwise contrasts. Contrasts were also performed with an equivalence testing approach and $90 \%$ confidence intervals. Equivalence bands were determined from reliability data for MVC taken from our laboratory using the same set-up as in the present study. The half-width of the minimal detectable change \% (6.98\%) was used. Because we did not have similar reliability data for TTF we opted to use the same relative minimal detectable change value for this dependent variable also in setting equivalence bands. The absolute equivalence bands for MVC and TTF were $\pm 6.45 \mathrm{Nm}$ and \pm 14.25 seconds respectively. For RPE and RPD we used the half-width of the minimal detectable change determined from previously published reliability data for these scales (Steele, et al., 2017). The absolute equivalence bands for RPE and RPS were \pm 1.18 and \pm 1.40 respectively. All tests were conducted with $\alpha=0.05$ for determination of statistical significance. In addition, data visualisation included plotting individual raw data and estimated marginal means for repeated measures between condition, in addition to pairwise contrasts with both $95 \%$ and $90 \%$ confidence intervals and equivalence bands. Standardised effect sizes were calculated as Cohen's $d$ (1992), and interpreted using Cohen's thresholds (>0.2 to $<0.5$ 'small'; $>0.5$ to $<0.8$ 'moderate'; $>0.8$ 'large') using the eff_size() function in the emmeans package.

\section{Results}

Peer) reviewing PDF | (2020:02:45680:1:0:NEW 6 Apr 2020) 
$\left(F_{(1,54)}=1.431, p=0.2368\right)$ though there was a significant main effect of Load $\left(F_{(1,54)}=1.431, p<0.001\right)$ with greater

TTF for 30\% MVC (caffeine[30\% MVC] = 204.1 seconds [172.1 to 236.1 seconds], placebo[30\% MVC] = 178.2 seconds [146.2 to 210.2 seconds], caffeine[70\% MVC] $=65.3$ seconds [33.3 to 97.3 seconds], placebo[70\% MVC] = 62.1 seconds [30.0 to 94.1 seconds]). There was also no statistically significant interaction effect of Condition $\mathrm{x}$ Load $\left(F_{(1,54)}=0.870, p=0.355\right)$. Cohen's $d$ for the between condition contrast was $0.49[-0.17$ to 1.15$]$ in $30 \% \mathrm{MVC}$, and 0.06 [-0.59 to 0.71$]$ in $70 \%$ MVC. Equivalence testing also did not reveal a statistically significant effect for Condition either in $30 \% \mathrm{MVC}\left(t_{(54)}=0.679, p=0.750\right)$ or $70 \% \mathrm{MVC}\left(t_{(54)}=-0.640, p=0.262\right)$ for the pairwise equivalence between the conditions 
$\left(F_{(1,54)}=0.518, p=0.475\right)$ or of Load $\left(F_{(1,54)}=3.056, p=0.086\right)$, and no statistically significant interaction effort of

Condition $\times$ Load $\left(F_{(1,54)}=0.095, p=0.759\right)$ with RPE appearing similar across all trials (caffeine[30\% MVC] $=8.26$

[7.65 to 8.87], placebo [30\% MVC] $=8.00$ [7.39 to 8.61$]$, caffeine[70\% MVC] $=8.63$ [8.02 to 8.24$]$, placebo[70\%

$M V C]=8.53$ [7.92 to 9.14]). Cohen's $d$ for the between condition contrast was 0.24 [-0.42 to 0.89$]$ in $30 \%$ MVC, both $30 \% \operatorname{MVC}\left(t_{(54)}=-2.528, p=0.007\right)$ and $70 \% \operatorname{MVC}\left(t_{(54)}=-2.964, p=0.002\right)$ for the pairwise contrasts. Visual inspection of figure 3 reveals that across both 30\% MVC and 70\% MVC there was equivalence in RPE between caffeine and placebo. and 0.07 [-0.58 to 0.72 ] in $70 \%$ MVC. Equivalence testing revealed a statistically significant effect for Condition in the $70 \% \operatorname{MVC}\left(t_{(54)}=-2.572, p=0.006\right)$ but not $30 \% \operatorname{MVC}\left(t_{(54)}=-1.108, p=0.136\right)$ for the pairwise contrasts. Visual inspection of figure 4 reveals that for 70\% MVC there was equivalence in RPD between caffeine and placebo.

\section{*INSERT FIGURE 4 AROUND HERE*}

\section{Discussion}

The present study considered the effects of low doses of caffeine upon maximal force (MVC) and time to task failure (TTF) at 30\% and 70\% MVC in females. The aim of the study was to better understand whether low 
273 (TTF) and whether this was associated with an effect of reducing perceptions of effort or discomfort. To our

274 knowledge this is the first study to consider the impact of low doses of caffeine $\left(<2 \mathrm{mg}^{\left.-\mathrm{kg}^{-1}\right)}\right.$ upon MVC and TTF for

275 heavier- and lighter-loads in females. We believe our study represents an ecologically valid approach since people

276 typically consume a predetermined absolute dosage of caffeine (e.g. 2 x Pro-Plus tablets; equating to a total of

277 100mg caffeine) and rarely calculate a specific dosage based on their body mass. Our main findings were that

$278100 \mathrm{mg}$ caffeine $\left(\sim 1.5 \mathrm{mg} \cdot \mathrm{kg}^{-1}\right)$ supported an increase in strength, but not relative muscular endurance compared to

279 a placebo condition. These results are discussed in greater detail below.

280

281 MVC

Our analyses revealed a significant effect for caffeine upon MVC in line with previous research showing

283

284

285

286

287

288

289

290

291

292

293

294

295

296

297

298

positive effects upon strength as a result of caffeine ingestion (Grgic, Sabol et al. 2019c). Thus, even at low doses

caffeine may have the potential to positively impact maximal force production. However, whilst analyses did reveal a statistically significant effect (i.e. excluding a zero effect), the confidence intervals did not exclude the upper equivalence bands (Figure 1B) and therefore it is unclear how meaningful this increase in MVC is. However, the standardised effect size (Cohen's $d$ ) for the caffeine compared to placebo condition for MVC was moderate $(d=$ 0.645 [0.155 to 1.14$]$ ) which interestingly is larger than that reported in meta-analyses considered in a recent umbrella review (Grgic et al., 2019a). Though, the inclusion of the upper equivalence band and the point and interval estimates of prior meta-analyses in the confidence interval range for our estimate suggests that the true population effect may be lower and questions its meaningfulness. Further, prior research examining maximal strength in females has shown generally trivial to small effects at best (Goldstein et al., 2010; Sabblah et al., 2015). However, we should recognize that caffeine consumption in females might stimulate very individual responses to MVC based on both genetic factors and the use of an oral contraceptive pill (Guest et al. 2018; Abernethy and Todd 1985). Inspection of the individual participant data in Figure 1 suggests there was considerable interindividual variation. However, the extent to which this is true inter-individual variation in response to caffeine as opposed to merely test-retest variation is unclear and future research should consider employing replicated randomised cross-over designs to investigate this (Atkinson et al. 2019).

Peer) reviewing PDF | (2020:02:45680:1:0:NEW 6 Apr 2020) 
301 failure for the $30 \%$ MVC conditions compared to the $70 \%$ MVC conditions ( $30 \%$ MVC: caffeine $=204.1$ seconds,

302 placebo $=178.2$ seconds; $70 \%$ MVC: caffeine $=65.3$ seconds, placebo $=62.1$ seconds). The body of literature has

303 reported that when exercising with lighter loads a person can/must exercise for considerably longer to reach 304 momentary failure (Fisher and Steele 2017; Stuart et al. 2018). In contrast to previous research, our analyses revealed no statistically significant difference for TTF as result of caffeine compared to placebo ingestion. Grgic et on relative muscular endurance at $40 \% 1 \mathrm{RM}$ appeared to be larger in males compared with females. It is worth considering however that with increases in strength resultant from interventions such as resistance training, relative muscular endurance typically does not change (Fisher et al. 2020). In previous studies, strength is normally measured using $1 \mathrm{RM}$ on a separate day to the placebo and caffeine conditions and so where there are changes this may be due to increased strength from supplementation resulting in the load used for muscular endurance testing being relatively less. In the present study we tested MVC on the day of each condition and after supplementation. increased, the load used for muscular endurance testing was set relative to that MVC. descriptively the lighter load ( $30 \% \mathrm{MVC}$ ) condition appears to differ between caffeine and placebo conditions (204.1 \pm 97.32 seconds, and $160.95 \pm 81.31$ seconds, respectively). While this was not statistically significant (neither excluding the upper equivalence bands nor a zero effect), a mean time change from placebo to caffeine of $\sim 45$ seconds might be practically meaningful. The standardised effect size for TTF following caffeine ingestion compared to placebo for the lighter load condition was moderate $(d=0.489[-0.169$ to 1.146$])$. This effect size is not too dissimilar from those reported in other meta-analyses though again the interval estimate suggests it may

323 be lower (Grgic et al., 2019a). Warren et al. (2010) suggested that endurance improvements are the result of 324 greater muscle fibre recruitment in the caffeine condition. However, the moderate effect for caffeine in the low 
325 load condition (30\% MVC) was not evident in the higher load condition (70\% MVC). At heavier loads

326 electromyographic amplitude (a proxy for motor unit recruitment) is higher than at lighter loads (Fisher et al.

327 2017). As such, caffeine might be less likely to positively influence muscular endurance by motor unit recruitment

328 in a heavier load condition. In contrast, at lighter loads caffeine ingestion might positively influence motor unit

329 cycling and re-recruitment to sustain the force requirements, and thus enhance muscular endurance.

In addition, prior research has suggested that caffeine might impact on TTF due to its effects upon

perceived effort and/or perceived pain (Motl et al. 2003; Motl et al. 2006; Astorino et al. 2011; Duncan et al. 2013;

de Morree et al. 2014; Smirnaul et al. 2017). Previous studies comparing lighter- and heavier-load exercise to

momentary failure have reported similar values for effort, but higher values for discomfort for a lighter load

condition (Fisher and Steele 2017; Stuart et al. 2018). Indeed, improvements in measures of relative muscular

endurance have been speculated to be in part due to greater tolerance of perceived discomfort (Fisher et al. 2020).

Thus, any performance enhancement in lighter-compared to heavier-load exercise may be due to effects upon perceptual variables. As such we measured both perceived effort (RPE) and perceived discomfort (RPD). significant differences between caffeine and placebo conditions for RPE which, whilst not maximal in value (e.g. testing was statistically significant suggesting that the RPEs reported were equivalent across conditions and loads. 
351 effort and discomfort where previous studies have generally failed to differentiate between the two. Since caffeine

352 is shown to produce analgesic effects that may improve performance by reducing pain perception (Motl et al.

353 2003; Motl et al. 2006; Astorino et al. 2011; Duncan et al. 2013), our data might simply support that effort was

354 equivalent and unaffected by conditions independently of pain/discomfort perception. or placebo conditions) suggests that an isometric task performed for time to failure might differ in the perceived discomfort incurred in comparison to performing traditional repetitions (e.g. both concentric and eccentric muscle actions). Certainly, previous research has supported differences in perceived discomfort between heavier- and lighter-load exercise (Fisher and Steele 2017; Stuart et al. 2018). Fisher and Steele (2017) reported RPD values for dynamic knee extension exercise of 8.7 and 6.5 for lighter-load (50\%MVC) and heavier-load (80\%MVC), respectively. Stuart et al. (2018) reported values of 6.3 for heavier-load (80\%MVC) and 8.0 and 8.3 for lighter load (50\%MVC) for males and females, respectively. However, perceived discomfort values from the present study using an isometric task were more similar (CAFF=8.0 and PLA=7.2 for 30\%MVC and CAFF=7.5 and PLA=7.4 for 70\%MVC). Previous literature has reported significantly greater values for pain and exertion for an isometric task using 50\%MVC compared to a dynamic task using 75\%MVC when performed to momentary failure (Frey Law et al. 2010). This suggests the relationship for discomfort might be different for isometric compared to dynamic muscle actions. Nonetheless, despite not finding statistically significant differences for perceived discomfort, we also did not find that RPD was statistically equivalent in the lighter-load condition whereas it was for the heavier-load condition. The standardised effect size for comparison of RPD between caffeine and placebo in the lighter-load condition $(d=0.543[-0.110$ to 1.196$])$ was not too dissimilar form the standardised effect size found for TTF $(d=$ 0.489 [-0.169 to 1.146]). But, contrastingly to the anticipated analgesic effects of caffeine, this suggested that during the lighter-load condition participants rated higher perceptions of discomfort as a result of caffeine 
377 caffeine that results in improved endurance under conditions of higher discomfort (i.e. lighter-loads) but this

378 should be examined in further research.

379

384

385

386

387

Limitations

It would be remiss not to discuss the limitations of the present study. Primarily, we did not include a nonplacebo control condition and as such we cannot be certain that a placebo effect was not evident. In addition, we did not assess the effectiveness of blinding upon participants who might have experienced typical sensation of increased arousal following caffeine consumption compared to placebo. Indeed, Saunders et al. (2016) suggest that supplement identification can influence exercise performance. Furthermore, we did not measure blood caffeine concentrations and thus the provision of a specific dose $(100 \mathrm{mg})$ combined with the potentially different absorption rates as a result of oral contraceptive or genetic factors cannot be discounted. A further limitation is the sample size in our study; however, we have presented a larger participant number than many previous studies, and we believe the study design along with the sample size fairly represents the results obtained. Based on the contemporary thinking surrounding a gene-caffeine interaction (Guest et al. 2018), as well as the possibility that an oral contraceptive pill might impact the effects of caffeine upon the body (Abernethy and Todd 1985), future research might consider genetic testing as well as consideration of the menstrual cycle and contraceptive use; something which we failed to do. As such a larger sample size might become redundant in females where assessment of the effects of caffeine could be measured based on individual variables. Also, we accept that larger caffeine doses (3-6mg $\mathrm{kg}^{-1}$ or larger) might produce more notable positive effects upon strength (Pickering and Kiely 2019). However, our aim was not to identify optimal dose or current best practice, but rather fill a gap within the literature (Spriet 2014) by considering whether, and how, low caffeine dosage impacts strength and muscular endurance at heavier- and lighter-loads. In addition, as noted we did not measure RPE or RPD during the exercise bouts and thus the lack of effects at task failure may miss any effect on the exercise bout. Lastly, we did not measure other possible mechanisms through which the effects of caffeine are thought to impact maximal strength and muscular endurance. Thus, the effects upon MVC seen here may be due to unmeasured mechanisms such as blocking the binding of adenosine to $A_{1}$ and $A_{2 a}$ G-protein coupled receptor sites and increased sarcoplasmic

Peer) reviewing PDF | (2020:02:45680:1:0:NEW 6 Apr 2020) 
402 reticulum calcium release impacting upon the central nervous system and motor unit recruitment respectively

403 (Grgic et al., 2019b).

\section{Conclusions}

In the present study of females, we found a statistically significant effect of low dose caffeine (100mg, equating to $\sim 1.50 \mathrm{mg} \cdot \mathrm{kg}^{-1}$ ) upon knee extension MVC, though the meaningfulness of this performance enhancement is less clear. Further, we did not find statistically significant effects of low dose caffeine upon TTF, or RPE and RPD. Indeed, RPE was statistically equivalent for isometric knee extension to momentary task failure in all conditions examined, and RPD for the heavier-load condition. As discussed herein, the dosage used represents an ecologically valid approach (e.g. a specific absolute dosage rather than a personalised relative dose per kg of body mass), and further, is representative of typical caffeinated beverages. Since caffeine consumption can present with

412 some deleterious side effects and withdrawal symptoms, we suggest a low caffeine dose (similar to that found in 413 typical caffeinated beverages) might be suitable practical approach to attain enhancements in muscular strength, 414 though not relative muscular endurance. 
415

416

417

418

419

420

421

422

423

424

425

426

427

428

429

430

431

432

433

434

435

436

437

438

439

440

441

442

443

444

445

446

447

448

449

450

451

452

453

\section{References}

Abernethy, D.R., and Todd, E.L. 1985. Impairment of caffeine clearance by chronic use of low-dose oestrogencontaining oral contraceptives. Eur. J. Clin. Pharmacol. 28(4): 425-428.

Arazi, H., Hoseinihaji, M., and Eghbali, E. 2016. The effects of different doses of caffeine on performance, rating of perceived exertion and pain perception in teenagers female karate athletes. Braz. J. Pharm. Sci. 52(4): 685-692.

Astorino, T.A., Terzi, M.N., Roberson, D.W., and Burnett, T.R. 2011. Effect of caffeine intake on pain perception during high-intensity exercise. Int. J. Sports. Nutr. Metab. 21(1): 27-32.

Atkinson, G., Williamson, P., and Batterham, A.M. 2019. Issues in the determination of 'responders' and 'non-responders' in physiological research. Exp. Physiol. 104(8): 1215-1225.

Bates, D., Mächler, M., Bolker, B.M., and Walker, S.C. 2015. Fitting Linear Mixed-Effects Models Using Ime4. J. Stat. Softw. 67(1): 1-47.

Bazzucchi, I., Felici, F., Montini, M., Figura, F., and Sacchetti, M. 2011. Caffeine improves neuromuscular function during maximal dynamic exercise. Muscle. Nerve. 43(6): 839-844.

Bruce, C.R., Anderson, M.E., Fraser, S.F., Stepto, N.K., Klein, R., Hopkins, W.G., and Hawley, J.A. 2000. Enhancement of 2000-m rowing performance after caffeine ingestion. Med. Sci. Sports. Exerc. 32(11): 1958-1963.

Cesareo, K.R., Mason, J.R., Saracino, P.G., Morrissey, M.C., and Ormsbee, M.J. 2019. The effects of a caffeine-like supplement, TeaCrine ${ }^{\circledR}$, on muscular strength, endurance and power performance in resistance-trained men. J. Int. Soc. Sports. Nutr. 16(1): 47

Chiang, C.M., Ismaeel, A., Griffis, R.B., and Weems, S. 2017. Effects of Vitamin D Supplementation on Muscle Strength in Athletes A Systematic Review. J. Strength. Cond. Res. 31(2): 566-574.

Cohen, J. 1992. A power primer. Psychol. Bull. 112: 155-159.

Costill, D.L, Dalsky, G.P, and Fink, W.J. 1978. Effects of caffeine ingestion on metabolism and exercise performance. Med. Sci. Sports. 10(3): 155-158.

Davis, J.K., and Green, J.M. 2009. Caffeine and anaerobic performance: ergogenic value and mechanisms of action. Sports. Med. 39(10): 813-832.

de Morree, H.M., Klein, C., and Marcora, S.M. 2014. Cortical substrates of the effects of caffeine and time-on-task on perception of effort. J. Appl. Physiol. 117(12): 1514-1523.

Del Coso, J., Muñoz, G., and Muñoz-Guerra, J. 2011. Prevalence of caffeine use in elite athletes following its removal from the World Anti-Doping Agency list of banned substances. Appl. Physiol. Nutr. Metab. 36(4): 555561.

Doherty, M., and Smith, P.M. 2005. Effects of caffeine ingestion on rating of perceived exertion during and after exercise: a meta-analysis. Scand. J. Med. Sci. Sports. 15(2): 69-78.

Duncan, M.J., Stanley, M., Parkhouse, N., Cook, K., and Smith, M. 2013. Acute caffeine ingestion enhances strength performance and reduces perceived exertion and muscle pain perception during resistance exercise. Eur. J. Sport. Sci. 13(4): 392-399.

Fisher, J., Steele, J., and Smith, D. 2017. High- and Low-Load Resistance Training: Interpretation and Practical Application of Current Research Findings. Sports. Med. 47(3): 393-400.

Fisher, J.P., and Steele, J. 2017. Heavier and lighter load resistance training to momentary failure produce similar increases in strength with differing degrees of discomfort. Muscle. Nerve. 56(4): 797-803. 
454

455

456

457

458

459

460

461

462

463

464

465

466

467

468

469

470

471

472

473

474

475

476

477

478

479

480

481

482

483

484

485

486

487

488

489

490

491

492

493
Fisher, J.P., Steele, J., Androulakis-Korakakis, P., Smith, D., Gentil, P., and Giessing, J. 2020. The strength-endurance continuum revisited: a critical commentary of the recommendation of different loading ranges for different muscular adaptations. J. Trainol. 9: 1-8

Frey Law, L.A., Lee, J.E., McMullen, T.R., and Xia, T. 2010. Relationships between maximum holding time and ratings of pain and exertion for static and dynamic tasks. Appl. Ergon. 42(1): 9-15.

Goldstein, E., Jacobs, P.L., Whitehurst, M., Penhollow, T., and Antonio, J. 2010. Caffeine enhances upper body strength in resistance trained women. J. Int. Soc. Sports. Nutr. 7:18.

Grgic, J., Grgic, I., Pickering, C., Schoenfeld, B.J., Bishop, D.J., and Pedisic, Z. 2019a. Wake up and smell the coffee: caffeine supplementation and exercise performance-an umbrella review of 21 published meta-analyses. Br. J. Sports. Med. E-pub ahead of print

Grgic, J., Mikulic, P., Schoenfeld, B.J., Bishop, D.J., and Pedisic, Z. 2019b. The influence of caffeine supplementation on resistance exercise: A review. Sports. Med. 49:17-30

Grgic, J., Sabol, F., Venier, S., Mikulic, I., Bratkovic, N., Schoenfeld, B.J., Pickering, C., Bishop, D.J., Pedisic, Z., and Mikulic, P. 2019c. What Dose of Caffeine to Use: Acute Effects of 3 Doses of Caffeine on Muscle Endurance and Strength. Int. J. Sports. Physiol. Perf. E-pub ahead of print

Guest, N., Corey, P., Vescovi, J., and El-Sohemy, A. 2018. Caffeine, CYP1A2 Genotype, and Endurance Performance in Athletes. Med. Sci. Sports. Exerc. 50(8): 1570-1578.

Juliano, L.M., and Griffiths, R.R. 2004. A critical review of caffeine withdrawal: empirical validation of symptoms and signs, incidence, severity, and associated features. Psychopharmacology. 176(1): 1-29.

Motl, R.W, O'Connor, P.J., and Dishman, R.K. 2003. Effect of caffeine on perceptions of leg muscle pain during moderate intensity cycling exercise. J. Pain. 4(6): 316-321.

Motl, R.W., O'Connor, P.J., Tubandt, L., Puetz, T., and Ely, M.R. 2006. Effect of Caffeine on Leg Muscle Pain during Cycling Exercise among Females. Med. Sci. Sports. Exerc. 38(3): 598-604.

Nehlig, A. 1999. Are we dependent upon coffee and caffeine? A review on human and animal data. Neurosci. Biobehav. Rev. 23(4): 563-76.

Pasman, W.J., van Baak, M.A., Jeukendrup, A.E., and de Haan, A. 1995. The effect of different dosages of caffeine on endurance performance time. Int. J. Sports. Med. 16(4): 225-230.

Pickering, C., and Kiely, J. 2018. What Should We Do About Habitual Caffeine Use in Athletes? Sports. Med. 49(6): 833-842.

Pickering, C., and Kiely, J. 2019. Are low doses of caffeine as ergogenic as higher doses? A critical review highlighting the need for comparison with current best practice in caffeine research. Nutr. 67-68.

Sabblah, S., Dixon, D., and Bottoms, L. 2015. Sex differences on the acute effects of caffeine on maximal strength and muscular endurance. Comp. Exerc. Physiol. 11(2): 89-94.

Saunders, B., de Oliveira, L.F., da Silva, R.P., de Salles Painelli, V., Gonçalves, L.S., Yamaguchi, G., Mutti, T., Maciel, E., Roschel, H., Artioli, G.G., Gualano, B. 2017. Placebo in sports nutrition: a proof-of-principle study involving caffeine supplementation. Scand. J. Med. Sci. Sports. 27(11): 1240-1247.

Smirnaul, B.P.C., de Moraes, A.C., Angius, L., and Marcora, S.M. 2017. Effects of caffeine on neuromuscular fatigue and performance during high-intensity cycling exercise in moderate hypoxia. Eur. J. Appl. Physiol. 117(1): 27-38.

Smit, H.J., and Rogers, P.J. 2000. Effects of low doses of caffeine on cognitive performance, mood and thirst in low and higher caffeine consumers. Psychopharmacology. 152(2): 167-173.

Peer) reviewing PDF | (2020:02:45680:1:0:NEW 6 Apr 2020) 
494

495

496

497

498

499

500

501

502

503

504

505

506

507

508

509

510

511

512

Spriet, L.L. 2014. Exercise and Sport Performance with low doses of caffeine. Sports. Med. 44(Suppl2): S175-S184.

Steele, J., Fisher, J., McKinnon, S., and McKinnon, P. 2017. Differentiation between perceived effort and discomfort during resistance training in older adults: Reliability of trainee ratings of effort and discomfort, and reliability and validity of trainer ratings of trainee effort. J. Trainol. 6(1): 1-8.

Strain, E.C., Mumford, G.K., Silverman, K., and Griffiths, R.R. 1994. Caffeine dependence syndrome. Evidence from case histories and experimental evaluations. JAMA. 272(13): 1043-1048

Stuart, C., Steele, J., Gentil, P., Giessing, J., and Fisher, J.P. 2018. Fatigue and perceptual responses of heavier- and lighter-load isolated lumbar extension resistance exercise in males and females. PeerJ, 16:6:e4523

Tarnopolsky, M. A. 2008. Effect of caffeine on the neuromuscular system - potential as an ergogenic aid. Appl. Phys. Nutr. Metab. 33(6): 1284-1289.

Temple, J.L., and Ziegler, A.M. 2011. Gender differences in subjective and physiological responses to caffeine and the role of steroid hormones. J. Caff. Res. 1(1): 41-48.

Timmins, T.D., and Saunders, D.H. 2014. Effect of caffeine ingestion on maximal voluntary contraction strength in upper- and lower-body muscle groups. J. Strength. Cond. Res. 28(11):3239-3244.

Warren, G.L., Park, N.D., Maresca, R.D., McKibans, K.I., and Millard-Stafford, M.L. 2010. Effect of caffeine ingestion on muscular strength and endurance: a meta-analysis. Med. Sci. Sports. Exerc. 42(7): 1375-87.

Wiles, J.D., Bird, S.R., Hopkins, J., and Riley, M. 1992. Effect of caffeinated coffee on running speed, respiratory factors, blood lactate and perceived exertion during 1500-m treadmill running. Br. J. Sports. Med. 26(2): 116120. 
Figure 1

Peak Torque $(A)$ and difference in peak torque $(B)$ between caffeine and placebo conditions
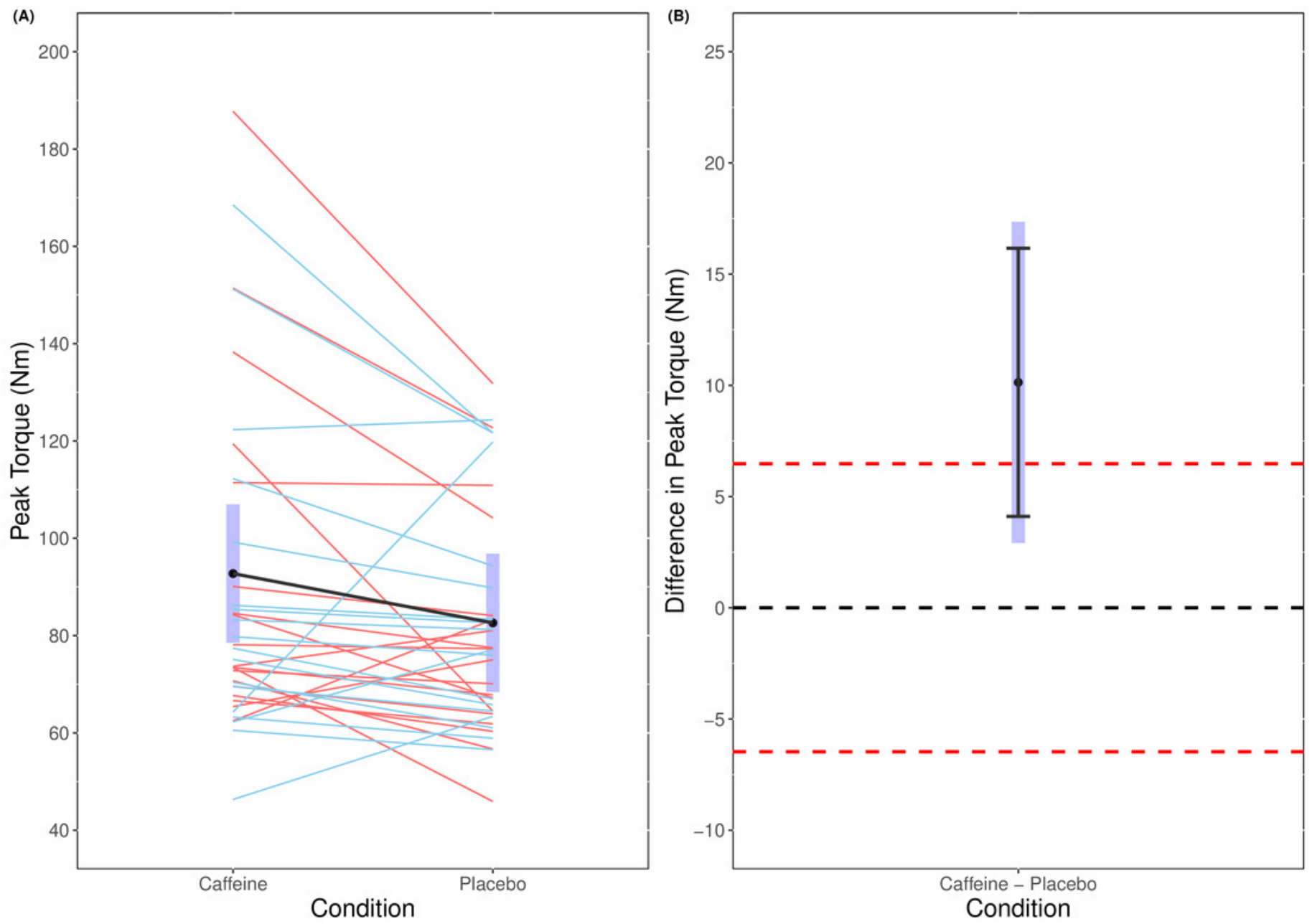
Figure 2

Time to task failure (A) and difference in time to task failure (B) for lighter (30\% MVC) and heavier (70\% MVC) load and between caffeine and placebo conditions
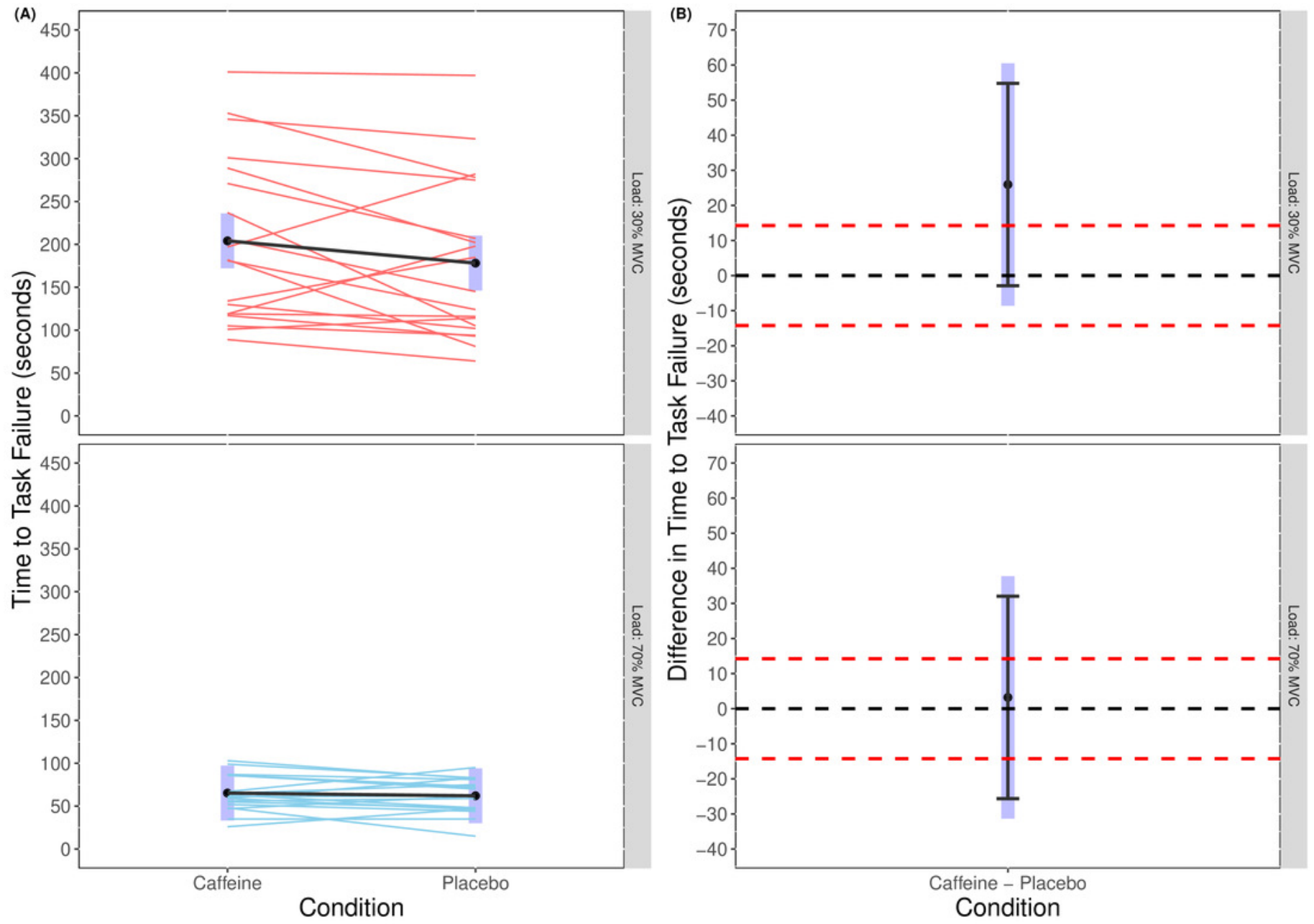
Figure 3

Rating of perceived effort (A) and difference in rating of perceived effort (B) for lighter (30\% MVC) and heavier (70\% MVC) load and between caffeine and placebo conditions
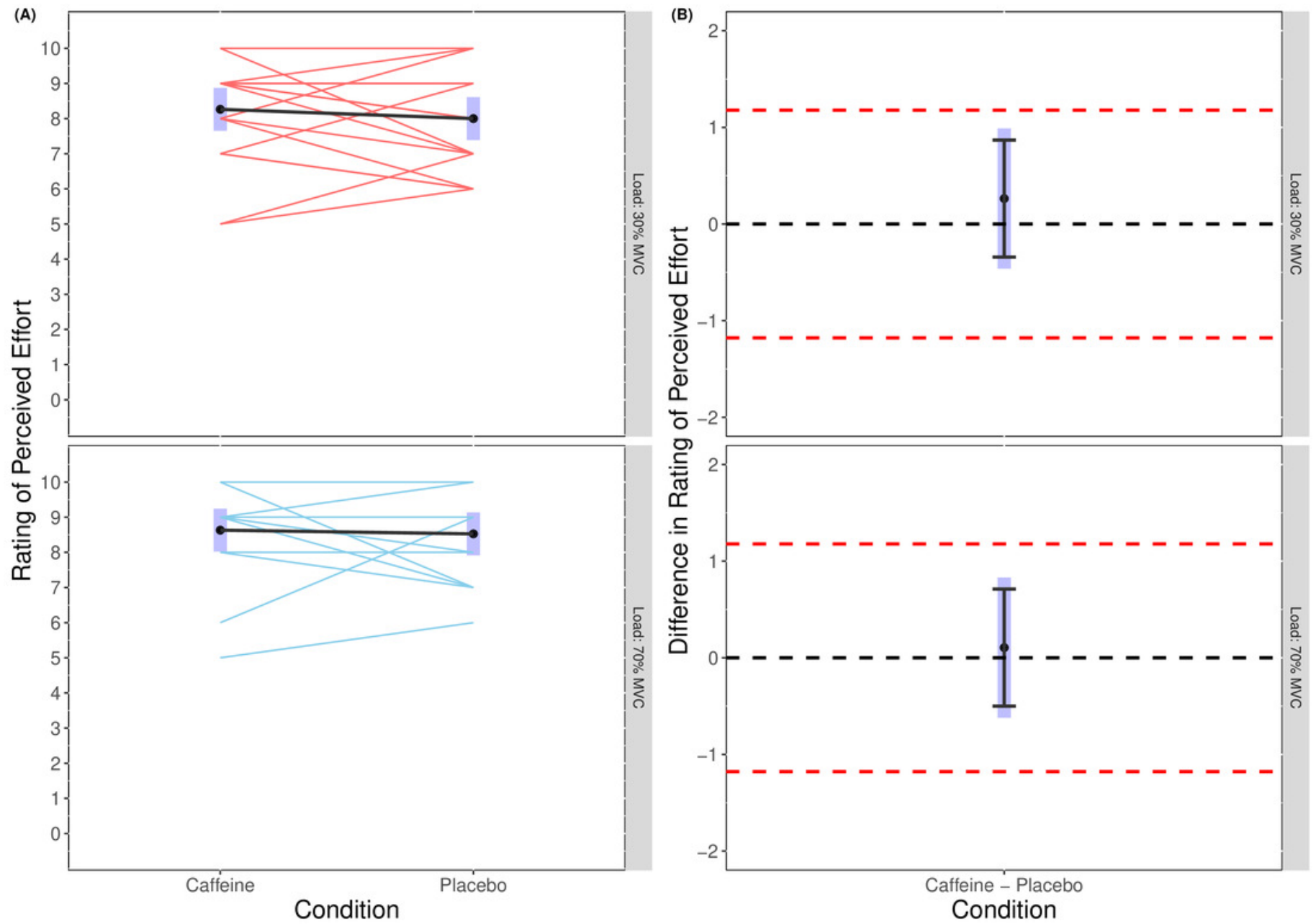
Figure 4

Rating of perceived discomfort $(A)$ and difference in rating of perceived discomfort (B) for lighter (30\% MVC) and heavier (70\% MVC) load and between caffeine and placebo conditions
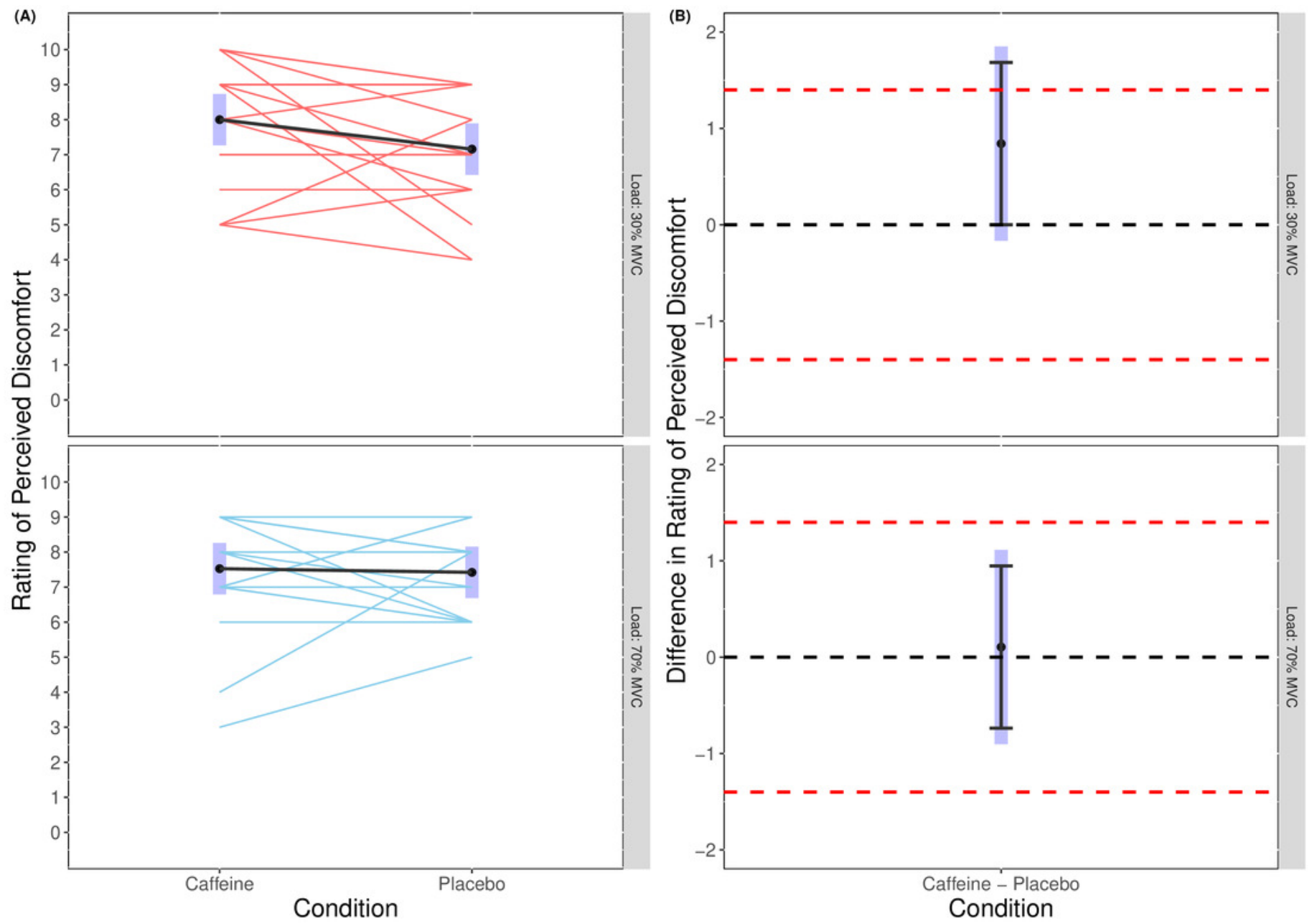